\title{
A 3.3 Gb/s Sample Circuit with GaAs MESFET Technology and SCFL Gates
}

Walter De Marco, Wilhelmus Van Noije* and Jacobus W. Swart

DSIF/FEEC/UNICAMP, Campinas, SP, Brazil "LSI/EPUSP, São Paulo, SP, Brazil

Av. Albert Einstein, 400

13081-970 Campinas - SP - Brazil

Phone : +55192398134 FAX: +55192391395

e-mail:Walter@dsif.fee.unicamp.br

\begin{abstract}
This paper presents the implementation of the sampling technique by means of the coordinated delays' method, using the SCFL (Source Coupled FET Logic) logic in GaAs MESFET technology. This technique presents a high resolution and is based on controlling delays in the clock and in the data signal paths, by means of delay elements. The resolution is related to the difference in the delay in both paths. The delay elements are implemented by means of differential invertors, in SCFL logic. A sample circuit of 64 stages has been designed. Its operation has been simulated using the HSPICE program that resulted in a high resolution at a bit rate of $3.33 \mathrm{~Gb} / \mathrm{s}$. The circuit prototypes are being fabricated at Vitesse Semiconductor, using the $\mathrm{H}-\mathrm{GaAs}$ III process with $0.6 \mu \mathrm{m}$ gate length. Measurement results "like maximum rate, resolution and jitter" will be presented at the meeting.
\end{abstract}

\section{Keywords}

Sampler, Coordinated delays, GaAs MESFET, SCFL Gates, High Speed Circuit 


\section{INTRODUCTION}

Sample circuits are used in many applications such as signal and clock recovery in data transmission systems, logic analyzers, measurement systems and many others. Higher resolutions and sampling rates are continuous goals for new generations and applications. Conventional circuits present limitations to sample high frequency signals, where enough samples per cycle have to be taken and setup and hold times also need to be taken into account. The appropriate distribution of the clock signal through the circuit is another design issue. To overcome these limitations two approaches can be adopted. One is to use high frequency device technology and the second is to use new circuit designs.

GaAs MESFET technology has become a mature and a viable option for high frequency needs. So, this technology was chosen for the design of our high bit rate sampling circuit.

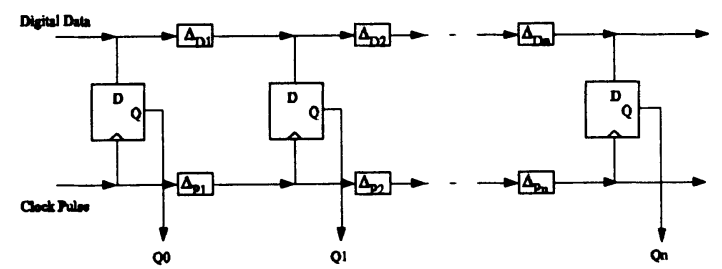

(a)

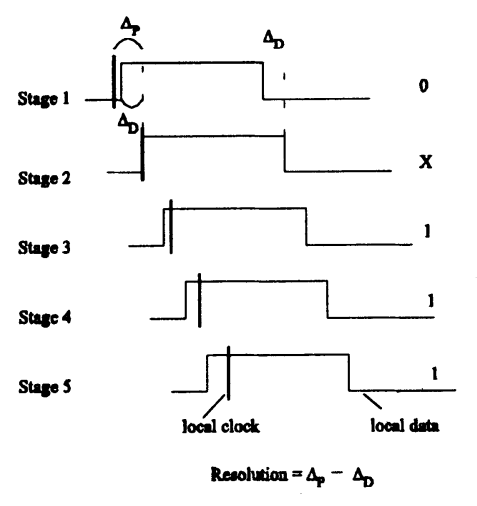

(b)

Figure 1 High Speed Digital Sampler: (a) circuits with delaying clock and data, (b) sampler operation. 
New sampling circuit techniques have been proposed to improve resolution by controlling the sampling time interval. This has been done trough the introduction of a controlled delay in the data path (Kim, 1990) or in the clock path (Bazers, 1992). It is shown that a better resolution can be obtained by means of introduction of simultaneous delays in both paths, data and clock, as illustrated in Figure 1 (Gray, 1994). The sampling time interval is given by the difference between the two delay elements, in the signal and clock paths. An additional advantage of the technique is that the sampling rate will be independent of the clock frequency.

A better understanding of the technique is obtained through the following equations:

$Q_{i-1}=D_{i-1}\left(T_{i-1}\right)$

$Q_{i}=D_{i}\left(T_{i-1}+\Delta_{P i}\right) \quad$ where $\mathrm{i}$ is an integer

$Q_{i}=D_{i-1}\left(T_{i-1}+\Delta_{P i}-\Delta_{b i}\right)$

The sampling interval is given by:

$\Delta_{P D i}=\left(\Delta_{P i}-\Delta_{D i}\right)$

where:

$Q_{i} \quad$ - output of latch i

$D_{i}(t)$ - digital wave signal at node $\mathrm{i}$

$T_{\text {clk }}$ - clock period time

$\Delta_{D i}$ - delay time of delay element $\mathrm{i}$ in signal path

$\Delta_{p i}$ - delay time of delay element $i$ in clock path

$T_{i} \quad$ - instant of sampling at node $i$.

If for every i, $\Delta_{D i}=\Delta_{D}$ and $\Delta_{P_{i}}=\Delta_{P}$, then the time difference between the delays of both elements will be constant with $\Delta_{P D}=\Delta_{P}-\Delta_{D}$. The digital signal will be sampled with a theoretical resolution given by $\Delta_{P D}$. The experimental minimum resolution can vary however at each node due to a spread of device parameter and due to variation of the operation ambient condition. This means that non linearities and non monotonicities in effective sampling times can occur (Gray, 1994). Special care has to be taken when drawing the circuit layout in order to minimize these effects. The resolution can be controlled and adjusted by means of adjusting the delay time of each element through an external signal.

The given sampling circuit presents a maximum frequency limit due to the fact that the delay elements and the latches present a low pass filter behavior. This 
means that a narrow input signal can not propagate through the sequence of stages of the circuit (Gray, 1994). The chosen GaAs MESFET technology and SCFL logic aim to extend the maximum signal frequency, compared to the previous CMOS technology circuit (Gray, 1994). This will allow the sampling of very narrow signal pulses. A second innovation is the use of differential inverters in both the delay stages as well as in the latches, which was not used in the CMOS case (Gray, 1994).

In this paper, a study on the implementation of the high frequency and high resolution sampling circuit is presented. A 64 stage circuit is designed with the HGaAs III P 0.6 $\mathrm{m}$ technology of Vitesse Semiconductor (Vitesse, 1993), accessed through the France CMP multi-project program. The HSPICE program is used to simulate the circuit (HSPICE, 1995). At the moment the prototype circuits are being fabricated. Measurement results will be presented at the conference.

\section{DESCRIPTION AND DESIGN OF THE SAMPLING CIRCUIT}

The sampling circuit is composed of three basic blocks, a delay unit for the clock signal, a delay unit for the data signal and the sampling latch unit. Figure 2 shows a block diagram of one of the 64 identical stages of the circuit.

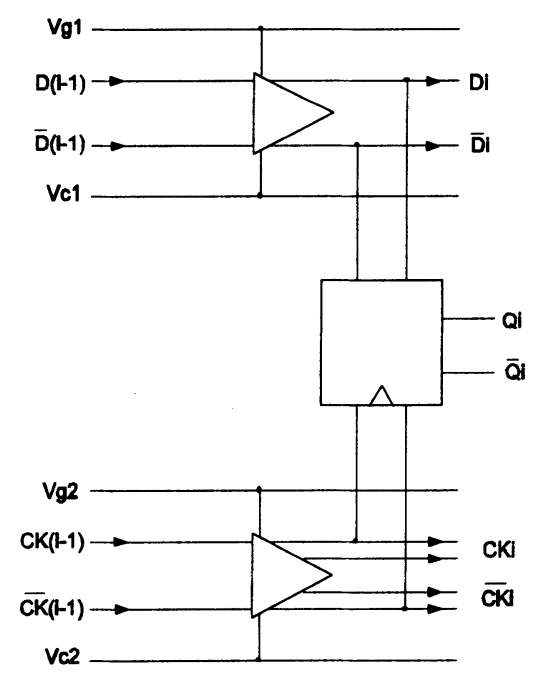

Figure 2 Block diagram of one stage of the sampler circuit.

A first issue in the design is to choose the type of basic inverters. DCFL (Direct-Coupled FET Logic) is the most simple logic gate for MESFET circuits (Long, 1990). Two other alternative gates suggested by the foundry are the SCFL (Source-Coupled FET Logic) and the BDCFL (Buffered Direct Coupled FET 
Logic). The DCFL and BDCFL gates present as advantages a lower power consumption and a smaller number of transistors. However, they suffer from high sensibility to threshold variations and from low fan-out capability (De Marco, 1996). The proposed sampling circuit needs a good control of delays that may consequently not vary with process and ambient variations. This imposes the use of less sensitive gates to these variations, as is the case of the SCFL gates due to its differential nature (Vu, 1988; De Marco, 1996). These differential gates are also less susceptible to noise, which will result in smaller jitter. This on its turn is of most importance to give a small (good) resolution of the sampler. Additionally, it offers a high fan-out capability, leading to smaller delays associated to interconnect line capacitances. These advantages of the SCFL gates are however paid by it's associated larger number of transistors, larger total area and larger power consumption.

Schematics of SCFL differential gates used as delay elements for the data signal and for the clock signal are shown in Figure 3 and Figure 4 respectively. One difference between them is the number of level shifter diodes at the output stage of the gates. As will be discussed below, the sample latch requires lower voltage levels for the clock signal than for the data signal. The resulting output swing of the gates is respectively $2.8 \mathrm{~V}$ to $3.5 \mathrm{~V}$ and $1.8 \mathrm{~V}$ to $2.5 \mathrm{~V}$, for a $\mathrm{V}_{\mathrm{DD}}$ bias of $5 \mathrm{~V}$ and with the gate bias terminals $\mathrm{V}_{\mathrm{G}}$ and $\mathrm{V}_{\mathrm{c}}$ connected to its respective transistor source. Figure 5 shows the transfer characteristic of the SCFL gate of Figure 3 (delay element of data signal). An average of $5 \mathrm{~mW}$ of power is consumed by each gate. The device dimensions were designed in order to: a) assure all transistors operate in the saturation region, to keep low gate-drain capacitances and b) assure low enough gate-source bias on the transistors, to limit its forward gate current. The output stage is included in each gate in order to adjust the appropriate output levels and to increase the fan-out driving capability. This last characteristic is adjusted by the width of the transistors of this output stage.

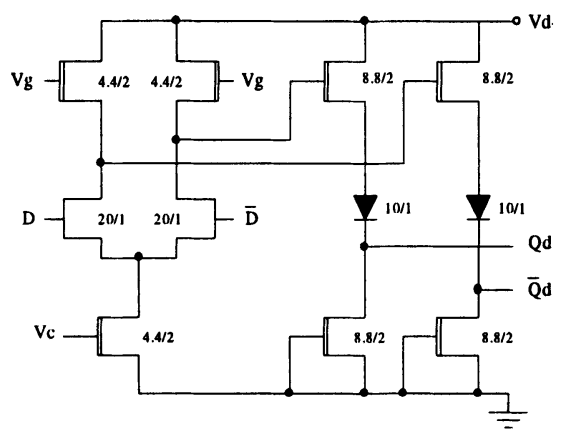

Figure 3 Schematic diagram of SCFL gate used as delay element for data signal. Channel dimensions, width / length, are indicated together to each transistor. 
The delay time of each gate can be adjusted by means of the applied biases at the transistor gates of the load transistors $\left(V_{G}\right)$ and the current sources $\left(V_{c}\right)$. The simulated results of delay times versus $V_{G}$ and $V_{c}$ are shown in Figure 6 for both gates of Figure 3 and Figure 4 . These results show that the bias $V_{c}$ presents a higher influence than $V_{G}$ on the delay time. This means that the delay depends mainly on the bias current trough the gate and less on the value of the load impedance. The results of Figure 6 also show that the delay of the clock signal invertors is larger than of the one of the data signal invertors. The larger delay of the clock signal gates is required in order to improve the resolution (Gray, 1994). Actually, the resolution (difference between both delays) can be adjusted by appropriately choosing the bias value of $\mathrm{V}_{\mathrm{G}}$ and $\mathrm{V}_{\mathrm{c}}$. This allows one to adjust it in accordance to the width of data signal.

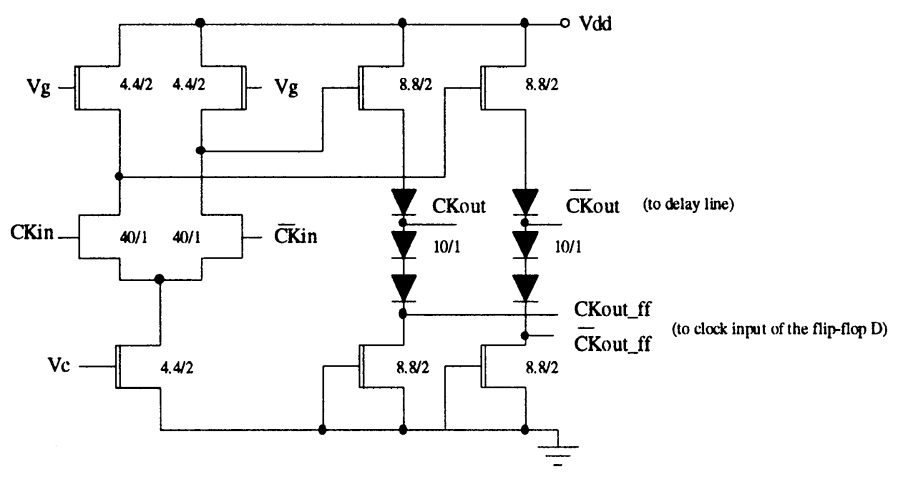

Figure 4 Schematic diagram of SCFL gate used as delay element for clock signal. Channel dimensions, width / length, are indicated together to each transistor.

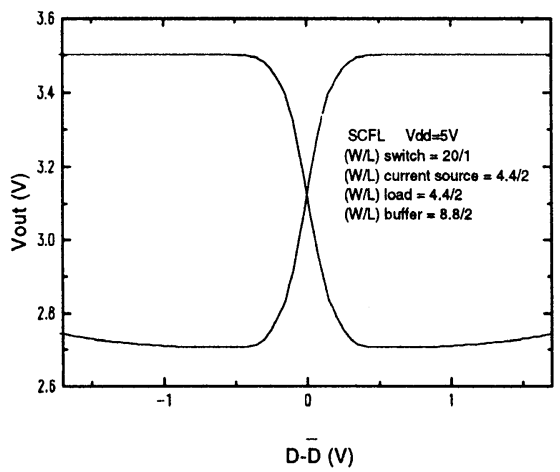

Figure 5 Transfer characteristic of the SCFL gate of Figure 3. 

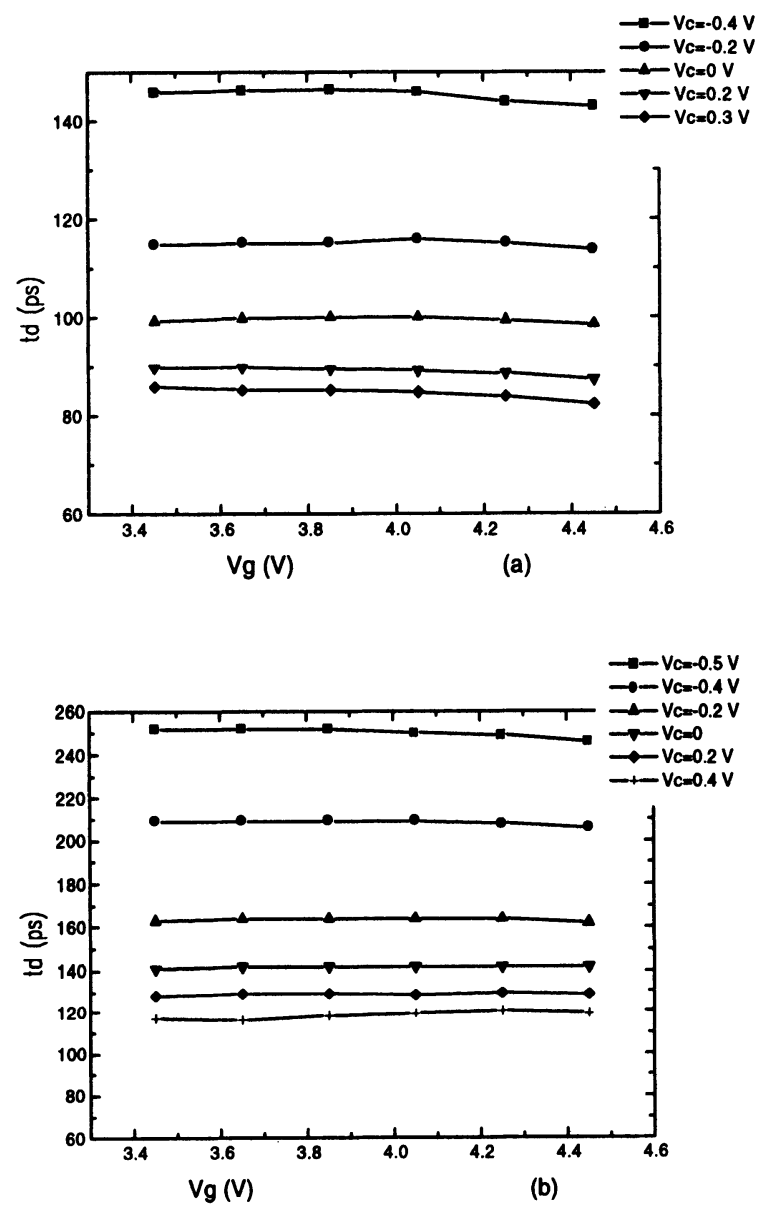

Figure 6 Simulated delay times versus bias $V_{G}$ and $V_{c}$ for the gates of (a) Figure 3 (data delay element) and (b) Figure 4 (clock delay element).

A D type SCFL flip-flop was used for the sampling latch. The schematic of the flip-flop is shown in Figure 7. This flip-flop is known as HLO-FO (High-Speed Latching Operation Flip-Flop) and presents approximately a 30\% improvement in speed compared to conventional latches (Murata, 1995). Its basic difference compared to conventional latches is that the read and latch stages have both different current paths. This allows an independent design for each to these stages. The use of this differential type of latch also avoids any possible metastability problem as described for the latch used in CMOS technology (Gray, 1994). Additionally, less noise or jitter is expected in the circuit once both clock and data signals are coming from differential type of delay lines. 


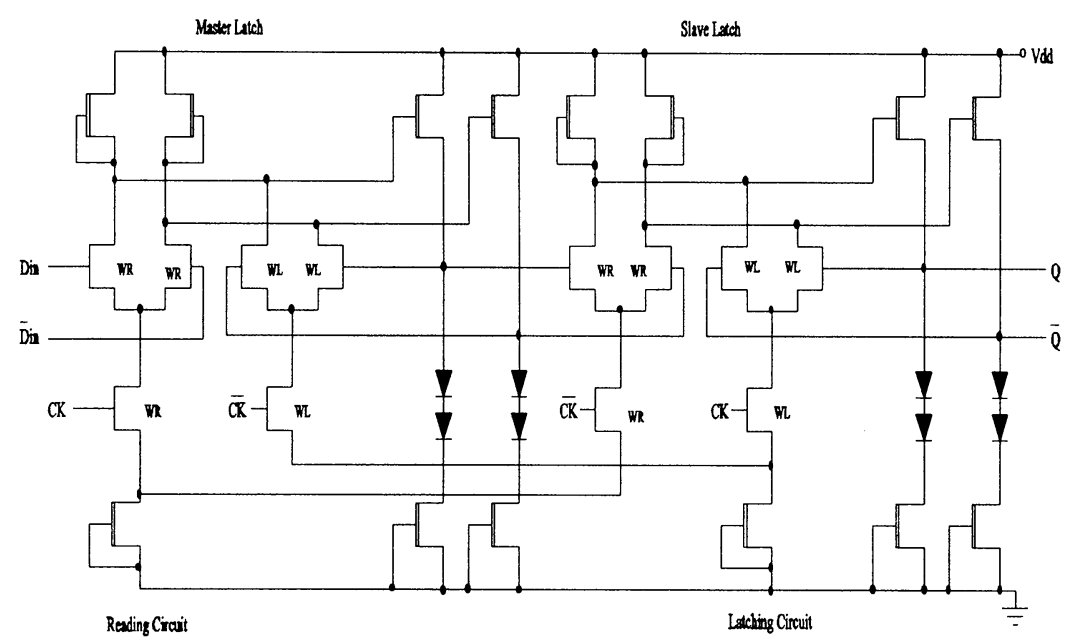

Figure 7 Schematic of the SCFL D type sampling flip-flop latch, known as HLO-FF.

\section{CIRCUIT LAYOUT AND RESULTS}

One important consideration to implement the layout is to take care to balance the capacitances at the output nodes of the differential gates and to make them equal for all the delay elements. This is needed in order to produce equal delay intervals for all the elements to reduce non linearity effects. Figure 8 shows the layout of one complete stage of the circuit, including the sampling D flip-flop and the data and clock signal delay elements. Three interconnect metallization levels are employed for routing and for power supply. Decoupling capacitors are added to the bias power lines. Additional ground lines are added in order to shield bias lines and reduce induced noise to the circuit. The layout of the complete stage is optimized in order to facilitate the cascading of the 64 stages of the complete circuit.

Figure 9 shows the layout of the complete test chip, including the 64 stage sampling circuit and two ring oscillators of 17 differential inverters. One ring oscillator is composed of inverters as used in the data signal delay elements (Figure 3) and the other one of inverters as used in the clock signal delay elements (Figure 4). The sampling circuit includes an output shift register of 64 bits. This avoids the need to connect output pads to all the 64 stages of the sampling circuit and will facilitate its testing. This shift register will be controlled by external clock signal. The signal transfer from the sampling stages to the shift register is performed through pass transistors enabled also by an external pulse. 


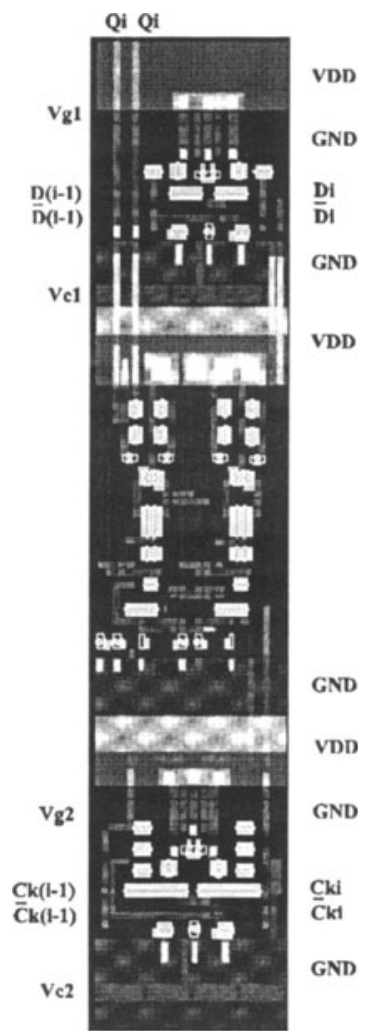

Figure 8 Layout of a single sampling stage of the circuit.

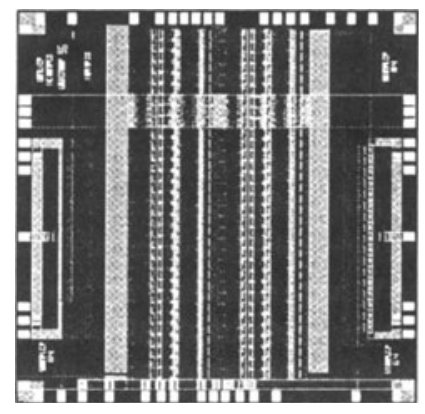

Figure 9 Layout of the complete test chip, including the 64 stage sampling circuit. 
HSPICE simulations of the circuit have been performed after layout parameter extraction, taking into account all parasitic interconnect line capacitances. Figure 10 shown a data string flowing through the signal path at the output of stage numbers 1,32 and 64 . This shows that a 300 ps width pulse can be sampled. The resolution of the sample circuit can theoretically vary from 0 to 185 ps. Experimentally, the minimum resolution will be larger than 0 ps due to the jitter effect. The maximum resolution value is determined by the difference between the maximum delay in the clock signal and the minimum delay in the data signal. Table 1 summarizes the present results and compares them with the ones obtained previously in CMOS technology (Gray, 1994).

An alternative design is presently being developed, aiming to reduce the chip area, improve the speed performance and reduce the power consumption at a low supply bias. This is being achieved by using the low-power enable-disable differential Logic (Ribas, 1996) and quasi-differentials switch flip-flop (Maeda, 1996), where the power supply can be brought below $1.0 \mathrm{~V}$.

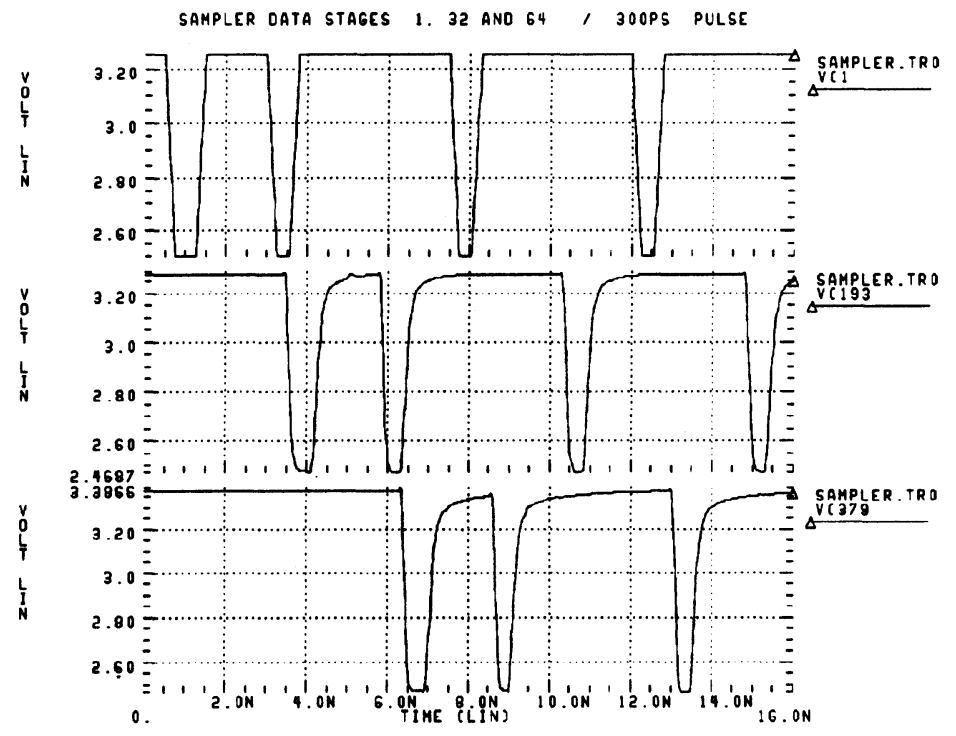

Figure 10 Simulated sampled data signal with 300 ps width pulses, at output of stages number 1,32 and 64 . 
Table 1 Design and performance of the sampler circuit in MESFET and its comparison to a previous CMOS circuit (Gray, 1994).

\begin{tabular}{lll}
\hline & MESFET & CMOS \\
\hline Transistor(sampler) & 3420 & 2624 \\
Die size & $4330 \mu \mathrm{m} \times 2100 \mu \mathrm{m}$ & $1795 \mu \mathrm{m} \times 3467 \mu \mathrm{m}$ \\
Total I/O pins & 28 (sampler) & 58 \\
Process & H-GaAs III P 0.6 $\mu \mathrm{m}$ Vitesse & MOSIS $1.2 \mu \mathrm{m}$ Nwell \\
Max. bandwidth & $3.33 \mathrm{~Gb} / \mathrm{s}$ & $1 \mathrm{~Gb} / \mathrm{s}$ \\
Resolution & $?-185 \mathrm{ps}$ & $25-250 \mathrm{ps}$ \\
Power dissipation & $2.5 \mathrm{~W}$ at $3.3 \mathrm{~Gb} / \mathrm{s}$ & $1 \mathrm{~W}$ at $1 \mathrm{~Gb} / \mathrm{s}$ \\
\hline
\end{tabular}

\section{CONCLUSIONS}

The design of a 64 stage sampling circuit based on the coordinated delay method, using SCFL gates on GaAs MESFET technology has been presented. The GaAs MESFET technology offers high frequency while the use of SCFL logic assures a robust and a metastability free sampling circuit. The sampling resolution can vary from a minimum to be determined experimentally to a maximum of 185 ps and the bandwidth is $3.3 \mathrm{~Gb} / \mathrm{s}$. The important measurement results of the sampler (resolution, maximum rate and jitter) will be presented at the conference.

\section{ACKNOWLEDGMENTS}

CNPq is acknowledged for scholarship, FAPESP for funding the chip fabrication and FINEP for its support in setting up the measurement facility. 


\section{REFERENCES}

Bazers, M. and Ashuri, R. (1992) "A novel CMOS digital and data decoder", IEEE Journal of Solid State Circuits, vol. 27, pp. 1934-1940.

De Marco, W. M. S., Custodio, R. M. F., Swart, J. W. and Van Noije, W. A. M. (1996) "Design and Analysis of DCFL and SCFL Logic Gates in GaAs MESFET technology", XI Conference of the Brazilian Microelectronics Society, Brazil, pp. 201-203.

Gray, T.G., Liu, W. and Van Noije, W. A. M. (1994) "A Sampling Technique and Its CMOS Implementation with $1 \mathrm{~Gb} / \mathrm{s}$ Bandwidth and 25ps Resolution ", IEEE Journal of Solid State Circuits, vol. 29, No. 3.

Hspice User's Manual (1995), (H92 version), Meta-Software, Inc.

Kim, B. , Helman, D.N. and P.R. Gray (1990) “A 30-MHz hybrid analog/digital clock recovery circuit in $2 \mu \mathrm{m}$ CMOS", IEEE Journal of Solid State Circuits, vol. 25, pp. 1385-1394 .

Long, S. I. and Butner, S. E. (1990) Gallium Arsenide Digital Integrated Circuit Design, McGraw-Hill.

Maeda, T., Numata, K., Fujii, M., Tokushima, M., Wada, S., Fukaishi, M. and Ishikawa, M. (1996) "An Ultra-Low-Power-Consumption High-Speed GaAs Quasi-Differential Switch Flip-Flop (QD-FF)", IEEE Journal of Solid State Circuits, vol. 31, No. 9, pp. 1361-1363.

Murata, K., Otsuji, T., Sano, E., Ohhata, M., Togashi, M. and Suzuk, M. (1995) "A Novel High-Speed Latching Operation Flip-Flop (HLO-FF) Circuit and its Application to a 19-Gb/s Decision Circuit Using a $0.2 \mu \mathrm{m} \mathrm{GaAs}$ MESFET", IEEE Journal of Solid State Circuits, vol. 30, No. 10, pp. 11011108.

Ribas, R.P., Bernal, A., Guyot (1996) "A Low-Power Enable/Disable GaAs MESFET Differential Logic”, GaAs IC Symposium Technical Digest, pp. 8184.

Vitesse Foundry Design Manual V. 6.0 (1993), Vitesse Semiconductor Corporation.

Vu, T., Peczalsky, A., Lee, K. W. and Conger, J. (1988) "The Performance of Source Coupled Fet Logic Circuits that Use GaAs Mesfets", IEEE Journal of Solid State Circuits, vol. 23, No. 1. 